\title{
Dossier \\ Phénotypage des animaux d'élevage
}

\section{Avant-propos}

La demande mondiale en produits d'origine animale est en forte expansion ${ }^{1}$ et l'élevage est l'une des activités agricoles les plus fortement créatrices d'emploi et de valeur ajoutée, tant au niveau de la production et des services qu'elle utilise, que des filières de produits animaux. Mais dans le même temps, l'élevage doit faire face à des enjeux sociétaux qui nécessitent des évolutions importantes dans les modes de production : besoin de compétitivité encore accru, méthodes d'élevage contestées vis-à-vis des atteintes à l'environnement, du respect du bien-être animal et de l'usage des médicaments. Il s'agit de réfléchir ces défis au niveau européen et mondial. Produire plus, mieux et à moindre coût doit contribuer à assurer la sécurité alimentaire mondiale et à répondre aux attentes européennes en termes de systèmes de production et de marchés très diversifiés. L'Europe a ici une responsabilité toute particulière car si elle ne peut pas nourrir le monde, il est de sa responsabilité, en tant que région où la demande sociale est aujourd'hui la plus élevée, de montrer comment concilier production et environnement.

Outre les innovations et les adaptations des systèmes d'élevage (cf. Numéro spécial de la revue «Quelles innovations pour quels systèmes d'élevage ? », Ingrand S., Baumont R. (Eds). INRA Prod. Anim., 27, 2), les réponses passent aussi par la recherche d'animaux qui soient d'une part, plus efficients pour transformer leur ration et plus faciles à élever dans une large gamme de conditions climatiques et de systèmes d'élevage et, d'autre part, adaptables face à des aléas climatiques, sanitaires ou économiques tout en préservant la santé, le bien-être et la qualité des produits. Par le passé, la recherche de la maximisation des performances de production (ex : vitesse de croissance, quantité de lait par lactation...) a conduit à des animaux de plus en plus spécialisés. Dans la plupart des filières, cette maximisation s'est accompagnée d'une dégradation des autres aptitudes d'élevage, aujourd'hui source d'inefficience en élevage. La recherche d'une plus grande robustesse (définie comme la capacité pour un animal d'exprimer son potentiel de production dans une large gamme d'environnements sans pour autant compromettre sa santé et son bien-être) devient une priorité. Dans le même temps, l'accès à des techniques d'exploration fonctionnelle toujours plus performantes, les innovations en cours d'appropriation ou à venir dans le domaine de la génomique ouvrent des perspectives d'investigation nouvelles plus précises.

Dans ce contexte d'évolution des demandes et des possibilités d'investigation, les projets de recherche en sciences animales doivent être plus systémiques, prédictifs et permettre d'établir des relations fonctionnelles de plus en plus fines entre le phénotype des animaux, leur génotype et le milieu dans lequel il s'exprime. Face au développement spectaculaire des connaissances sur le génome, il y a un manque criant de connaissances sur l'expression des phénotypes, connaissances qui devraient permettre de répondre au mieux à une double finalité en termes d'exploitation de la variabilité des aptitudes animales : $i$ ) une sélection éclairée vers des objectifs majeurs pour améliorer l'efficience de la production et la robustesse des génotypes, et ii) un élevage de précision qui valorise la variabilité individuelle des animaux pour gagner en efficience et en résilience à l'échelle du troupeau, ou pour améliorer la conduite des animaux d'un génotype donné. En effet, d'un coté les progrès réalisés par la sélection génomique permettent aujourd'hui de repenser les critères de sélection et de les diversifier tout en raccourcissant les délais entre la définition des objectifs et l'amélioration effective des cheptels, mais cette sélection entraine de nouveaux besoins de phénotypage car elle nécessite la caractérisation d'une

\footnotetext{
1 Selon la FAO et l'OCDE, la progression de la consommation de protéines d'origine animale sur la planète devrait se poursuivre au rythme de +2 à $+3 \% /$ an au cours de cette décennie. Sur la période 2000-2050, la consommation totale des viandes devrait progresser de l'ordre de $+70 \%$ pour une population en augmentation de $+20 \%$. Selon les mêmes sources et pour la même période la consommation de lait et produits laitiers dans les pays émergents devrait passer de 45 à $78 \mathrm{~kg}$ par habitant.
} 
population de référence. D'un autre coté, la connaissance plus fine de l'animal associée aux technologies en émergence de l'élevage de précision permettra de mieux piloter la conduite d'élevage pour améliorer l'efficience de l'alimentation ou de la reproduction par une approche individuelle à l'animal, ainsi que par la production d'alertes à destination de l'éleveur pour un diagnostic précoce des troubles permettant d'anticiper les ajustements requis.

Le phénotypage est ainsi l'un des principaux défis que doit relever les recherches en production animale et a été reconnu comme tel par l'INRA. Deux types de phénotypage peuvent être envisagés : le phénotypage des caractères d'intérêt socio-économique et le phénotypage de caractères plus élémentaires. Les phénotypes d'intérêt socio-économique constituent la finalité recherchée. Ils résultent de mécanismes faisant appel à des régulations complexes, ils sont d'autant plus onéreux à mesurer qu'il s'agit de critères zootechniques qui doivent être observés sur des pas de temps longs. La recherche de phénotypes plus élémentaires et plus proches du mécanisme causal rend plus facile l'identification des gènes responsables. Ce phénotypage fin implique de réaliser des mesures particulièrement approfondies et à des échelles élémentaires (au niveau moléculaire, cellulaire, tissulaire...) des caractéristiques biologiques de l'animal qui expliquent un phénotype complexe observé à l'échelle de l'animal. Le phénotypage à haut débit signifie qu'une méthode de mesure des phénotypes fiable, automatisable et rapide est établie de sorte que le processus de mesure permette de générer un grand nombre de données dans un laps de temps court. Le haut débit peut s'appliquer au phénotypage fin tout comme à celui des caractères d'intérêt zootechnique. Les contributions significatives qui pourront être attendues d'un phénotypage animal fin et à haut débit concernent la biologie prédictive, à savoir la prédiction des performances à partir de caractères simples observables précocement, nécessaire notamment à la gestion des produits, à la conduite de l'élevage et à la sélection animale.

Ce dossier propose le fruit d'une réflexion globale des chercheurs de l'INRA sur les perspectives qu'offre le phénotypage des animaux pour répondre aux enjeux des productions animales. Cette réflexion a eu pour objectif de définir les grands enjeux de connaissance autour du phénotypage animal et de faire émerger les questions de recherches prioritaires, transversales ou spécifiques aux différentes filières animales ainsi que les verrous techniques qui sont soulevés. Cette réflexion a été conduite par un groupe de douze chercheurs ${ }^{2}$ des départements de " Génétique Animale », "Physiologie Animale et Systèmes d'Elevage » et "Santé Animale » travaillant dans des disciplines et sur des espèces variées, missionné par la Direction Scientifique Agriculture de l'INRA. La réflexion de ce groupe s'est appuyée sur celle d'un collectif plus large de chercheurs INRA travaillant sur les animaux d'élevage par le biais d'un séminaire organisé en mai 2013 ainsi que par la rédaction partagée des articles de ce dossier.

L'amélioration de la robustesse des animaux d'élevage étant un objectif central, le premier article de ce dossier développe une approche systémique de la robustesse des animaux d'élevage pour répondre au mieux à une double finalité en termes d'exploitation de la variabilité des aptitudes animales pour la sélection et pour la conduite de l'élevage. Les recherches sur le phénotypage doivent être accompagnées d'une ontologie générique c'est-à-dire d'une représentation et d'un langage communs pour partager les données et les connaissances, ainsi que l'explicite le second article de ce numéro. L'objet des trois synthèses suivantes est de mettre en perspective les fronts de science (phénotypage du microbiote intestinal), les enjeux de connaissance et les verrous techniques encore à lever pour permettre des productions animales de qualité (phénotypage de la qualité des produits) et en quantité (phénotypage de l'efficacité alimentaire), à moindres coûts économique et environnemental.

Jean-Louis Peyraud

Inra, Direction Scientifique Agriculture Chargé de mission
Florence Phocas

Inra, UMR Génétique Animale et Biologie Intégrative Directrice de Recherche

\footnotetext{
2 Julien Bobe, Loys Bodin, Bernard Charley, Jean-Yves Dourmad, Nicolas Friggens, Jean-François Hocquette, Pierre-Yves Le Bail, Elisabeth Lebihan-Duval, Pierre Mormède, Florence Phocas (animatrice), Pascale Quéré et François Schelcher.
} 